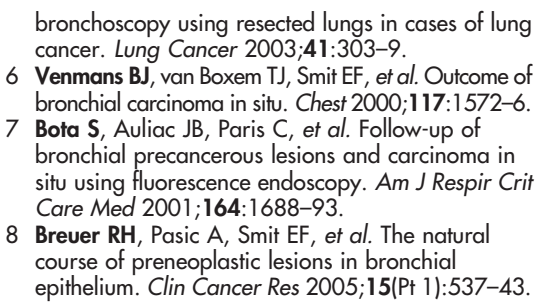

bronchoscopy using resected lungs in cases of lung cancer. Lung Cancer 2003:41:303-9.

6 Venmans BJ, van Boxem TJ, Smit EF, et al. Outcome of bronchial carcinoma in situ. Chest 2000;1 17:1572-6.

7 Bota S, Auliac JB, Paris C, et al. Follow-up of bronchial precancerous lesions and carcinoma in situ using fluorescence endoscopy. Am J Respir Crit Care Med 2001; 164:1688-93.

8 Brever RH, Pasic A, Smit EF, et al. The natural course of preneoplastic lesions in bronchial epithelium. Clin Cancer Res 2005;15(Pt 1):537-43.

9 Lam S, Slivinskas J, McWilliams A, et al. Natural history of premalignant bronchial lesions: implications for chemoprevention. Am Assoc Cancer Res 2002.

10 George PJ, Banerjee AK, Read CA, et al. Surveillance for the detection of early lung cancer in patients with bronchial dysplasia. Thorax 2005;62:43-50.

11 Cortese DA, Edell ES, Kinsey J. Photodynamic therapy for early stage squamous cell carcinoma of the lung. Mayo Clin Proc 1997;72:595-602.
12 Edell ES, Cortese DA. Bronchoscopic phototherapy with hematoporphyrin derivative for treatment of localized bronchogenic carcinoma: a 5 -year experience. Mayo Clin Proc 1987:62:8-14

13 Kato H. Photodynamic therapy for lung cancer of-a review of 19 years experience. J Photchem Photobiol 1998:42:96-9.

14 Mathur PN, Edell ES, Sutedja T, et al. Treatment of early stage non-small cell lung cancer. Chest 2003;123:S176-80.

\section{What is this thing called CFA?}

\section{A U Wells, D M Hansell, A G Nicholson}

\section{The term "cryptogenic fibrosing alveolitis"should now be used as strictly synonymous with "idiopathic pulmonary fibrosis"}

\footnotetext{
A rcane diagnostic labels bother clinicians. The diffuse lung disease lexicon is a notorious example. For decades, "diffuse lung disease speak" consisted of an unholy mix of histopathological and clinical terms, varying between countries, within countries and even between medical teams in the same hospital. Radical change was required and proposals were advanced in a joint American Thoracic Society and European Respiratory Society (ATS/ERS) initiative. Terminology for idiopathic interstitial pneumonia was distilled by a core group of clinicians, radiologists and pathologists, and this was then circulated to a larger group of international reviewers and published in 2002. ${ }^{1}$ The final consensus classification was not, at first sight, straightforward to apply. Indeed, the amorphous entity of "non-specific interstitial pneumonia" (NSIP) continues to vex clinicians and requires further subclassification. However, teething problems aside, the ATS/ERS initiative has been an outstanding success. Clinicians and researchers worldwide now understand each other better than before. The recent move towards large multicentre treatment studies in idiopathic pulmonary fibrosis (IPF), itself a revolution in slow motion, was made possible, in no small part, by this standardisation of terminology and disease definitions.

As the terminology has changed, the article by Rudd et $a l^{2}$ in the current issue of Thorax (see $p$ 67) is likely to pose difficulties for some non-UK readers. The authors have studied "cryptogenic fibrosing alveolitis" (CFA) as a clinical presentation, as used historically in the UK. The diagnostic criteria, consisting of
}

compatible radiographic, pulmonary function and clinical findings, in the absence of an overt environmental or autoimmune cause, are highly non-specific: compatible, also, with idiopathic interstitial pneumonias other than IPF and a subgroup of patients with hypersensitivity pneumonitis. By contrast, in the ATS/ERS classification, ${ }^{13}$ CFA is explicitly synonymous with IPF, as defined at biopsy or using high resolution computed tomography (HRCT) criteria, with typical clinical features also required, and this is now accepted internationally. Rudd et $a l^{2}$ discuss this issue in their methods section. However, their definition of CFA, as corresponding to "idiopathic pulmonary fibrosis in US terminology", is no longer correct using the current classification and, in reality, was probably never correct. In historical US series, the diagnosis of IPF was wholly or largely based on histopathological data; the application of ATS criteria essentially involved reclassifying NSIP cases and a small number of other disorders. ${ }^{4}$ By contrast, the historical UK entity of CFA was diagnosed at surgical biopsy in only $12 \%$ of cases in the early 1990s, ${ }^{5}$ and a large number of disorders other than IPF were necessarily included. Thus, the current British Thoracic Society (BTS) study is nothing more or less than a study of a non-specific clinical presentation, and unless this is understood, the findings will be misinterpreted.

The presence of two very different entities in the current medical literature, both termed CFA, cannot be a good thing. However, in their use of historical terminology, Rudd et al ${ }^{2}$ perhaps imply that something nosologically important has been lost. The concept of a "CFA" presentation is undoubtedly useful. The typical clinical picture is readily recognised in the outpatient clinic, even before tests such as HRCT. A "CFA" presentation is a key starting point in the personal diagnostic algorithms of many experienced clinicians. Furthermore, knowledge of a clinical picture of "CFA" informs prognostic evaluation. It is increasingly clear that the histological pattern of NSIP is associated with several distinct clinicoradiological entities, with HRCT and clinical findings often those of organising pneumonia with fibrosis ${ }^{6}$ or hypersensitivity pneumonia. ${ }^{7}$ However, the good outcome in these subgroups contrasts with outcomes in patients with idiopathic NSIP with a "CFA" clinical presentation: although prolonged survival is sometimes attainable, a poor IPF-like outcome is equally common, despite aggressive treatment. ${ }^{8}$ A frank admission that the prognosis is uncertain is crucial if therapeutic options are to be discussed with patients in an informed manner.

Perhaps more importantly, a "CFA" presentation has been, and is, indispensable for epidemiological work. It is simply unrealistic to require the application of formal ATS/ERS criteria for IPF, including HRCT, biopsy and bronchoalveolar lavage findings, in studies of the prevalence and spectrum of disease outside referral centres. An inclusive approach is required-in other words, a clinical diagnosis is necessary. Epidemiological work on diffuse lung disease is vital. The track record of public funding bodies (such as the Medical Research Council) in supporting therapeutic studies of diffuse lung disease is poor, partly because of a lingering perception that IPF is an uncommon disease confined to referral centres. Studies to establish the true impact of diffuse lung disease outside specialist units are particularly important in disorders that are strikingly more prevalent with increasing age, but this view was, perhaps, underrepresented in the ATS/ERS deliberations. The redefinition of CFA as synonymous with IPF has effectively disenfranchised epidemiologists, who, it could be argued, now have to study a disorder without a 
name. It is hardly surprising that some researchers in the field are disenchanted with the ATS/ERS classification and cling to the terminology of the past century.

However, the needs of epidemiologists are sometimes trumped by clinical imperatives. Diagnostic separations are valuable when they provide useful distinctions in the natural history and treated course of disease. The ATS/ERS consensus classification passes this test with flying colours: fundamentally, diagnosis is prognosis. In a study of consecutive patients with a "CFA" presentation during the 1980s, who were considered to have CFA at biopsy, reclassification resulted in three broad patient groups. ${ }^{8}$ Histological patterns of usual interstitial pneumonia (denoting IPF), fibrotic NSIP and a grouping of predominantly inflammatory disorders, seen in $45 \%, 35 \%$ and $20 \%$ of patients, were associated with 6-year survival rates of $10 \%, 50 \%$ and $100 \%$, respectively. It is difficult to envisage a more definitive prognostic separation. Thus, the application of the ATS/ERS system identifies separate patient subsets with survival rates broadly similar to those of patients with lung cancer, breast cancer and basal cell carcinoma of the skin. Thus far, cancer specialists have resisted the temptation to amalgamate these into "cryptogenic cancer". In diffuse lung disease, as in oncological practice, diagnostic distinctions can sometimes be difficult in individual patients, with interobserver and intraobserver diagnostic variation, ${ }^{9}{ }^{10}$ and diagnostic overlap between the classic entities, ${ }^{11}$ both recognised problems. However, even without good understanding of the entity with which one is dealing, diagnostic distinctions are worth pursuing because they allow informed discussion of treatment goals as well as accurate management. In idiopathic interstitial pneumonia, accurate diagnosis, which can often be based on appearances on HRCT rather than histopathological findings, distinguishes between inexorably progressive fibrosis (IPF), indolent fibrosis that sometimes has a good treated outcome (fibrotic NSIP) and the remaining inflammatory disorders that often do well with relatively restrained levels of treatment. To fail to make these distinctions is to play dice with patient management. "Presentation with CFA" is no longer an acceptable final diagnosis.
The counter-argument is that idiopathic interstitial pneumonias other than IPF are vanishingly rare in non-referral populations, and therefore have little or no relevance in routine practice. This contention, used to justify retention of the term "CFA" in its historical sense, is based on the more advanced age of nonreferral populations and the observation of a somewhat lower prevalence of NSIP in older patients in some series from specialist centres. However, the trend has not, in reality, been properly quantified and there is a pressing need for further studies of this question in unselected populations, perhaps based on appearances on HRCT rather than histopathological findings. The data of Rudd et $a l^{2}$ are instructive because they provide strong indirect evidence that a substantial minority of patients with "CFA" do, in fact, have disorders other than IPF. A response to treatment, as judged by marked increases in pulmonary function indices, was seen in $35 \%$ of patients, a figure that uncannily resembles the response rate reported in the histopathological study discussed earlier, ${ }^{8}$ in which $<50 \%$ of patients had IPF. By contrast, responsiveness seldom exceeds 10\% in studies of IPF in the post-ATS/ERS classification era The mortality data in the BTS study is also persuasive. Subsequent to the ATS ERS classification, outcomes of patients treated for IPF have been evaluated against age stratification in only one large cohort $^{12}$; in patients aged $\geqslant 70$, the 4-year survival of $15 \%$ is substantially lower than the 4-year survival of $40 \%$ in the BTS cohort. Taken together, the response rates and mortality data in the BTS study are highly suggestive of a large subgroup of patients with interstitial pneumonias other than IPF (including undiagnosed hypersensitivity pneumonia), who are not best served by an indiscriminate final diagnosis of "CFA".

It is unrealistic to hope to rehabilitate historical terms when a new classification is, in effect, written in marble-minor refinement aside, the ATS/ERS classification of the idiopathic interstitial pneumonias is here to stay. For clarity of thought, the term "CFA" should now be used as strictly synonymous with IPF, as currently defined using HRCT or biopsy criteria, in the correct clinical context. The creation of a new term such as "CFA clinical syndrome", for use by epidemiologists in studies of pulmonary fibrosis, is now absolutely necessary. It is time to move on.

Thorax 2007:62:3-4.

doi: $10.1136 /$ thx.2005.051011

\section{Authors' affiliations \\ A U Wells, D M Hansell, A G Nicholson, Royal Brompton Hospital, London, UK}

Correspondence to: $\operatorname{Dr} \mathrm{A} U$ Wells, Royal Brompton Hospital, Sydney Street, London SW3 6NP, UK; a.wells@rbh.nthames.nhs.uk

Competing interests: None declared.

\section{REFERENCES}

1 American Thoracic Society, European Respiratory Society. American Thoracic Society/European Respiratory Society international multidisciplinary consensus classification of the Idiopathic Interstitial Pneumonias. Am J Respir Crit Care Med 2002; 165:277-304

2 Rudd R, Prescott RJ, Chalmers JC, et al. British Thoracic Society Study of cryptogenic fibrosing alveolitis: response to treatment and survival. Thorax, this issue.

3 American Thoracic Society. Idiopathic pulmonary fibrosis: diagnosis and treatment. International consensus statement. Am J Respir Crit Care Med 2000;161:646-64.

4 Bjoraker JA, Ryu JH, Edwin MK, et al. Prognostic significance of histopathological subsets in idiopathic pulmonary fibrosis. Am J Respir Crit Care Med 1998; 157:199-203.

5 Johnston ID, Prescott RJ, Chalmers JC, et al. British Thoracic Society study of cryptogenic fibrosing alveolitis: current presentation and initial management. Fibrosing Alveolitis Subcommittee of the Research Committee of the British Thoracic Society. Thorax 1997;52:38-44.

6 Nagai S, Kitaichi $M$, Itoh $\mathrm{H}$, et al. Idiopathic nonspecific interstitial pneumonia/fibrosis: comparison with idiopathic pulmonary fibrosis and BOOP. Eur Respir J 1998;12:1010-19.

7 Selman M, Pardo A, Barrera L, et al. Gene expression profiles distinguish idiopathic pulmonary fibrosis from hypersensitivity pneumonitis. Am J Respir Crit Care Med 2006;173:188-98.

8 Nicholson AG, Colby TV, du Bois RM, et al. The prognostic significance of the histologic pattern of interstitial pneumonia in patients presenting with the clinical entity of cryptogenic fibrosing alveolitis. Am J Respir Crit Care Med 2000;162:2213-17.

9 Nicholson AG, Addis BJ, Bharucha $\mathrm{H}$, et al. Interobserver variation between pathologists in diffuse parenchymal lung disease. Thorax 2004:59:500-5.

10 Aziz ZA, Wells AU, Hansell DM, et al. HRCT diagnosis of diffuse parenchymal lung disease: inter-observer variation. Thorax 2004;59:506-11.

11 Flaherty KR, Thwaite EL, Kazerooni EA, et al. Radiological versus histological diagnosis in UIP and NSIP: survival implications. Thorax 2003;58:143-8.

12 King TE, Tooze JA, Schwarz MI, et al. Predicting survival in idiopathic pulmonary fibrosis: scoring system and survival model. Am J Respir Crit Care Med 2001; 164:1171-81. 